\title{
Powers of Complex Persymmetric Antitridiagonal Matrices with Constant Antidiagonals
}

\author{
Haibo Wang \\ College of Science, University of Shanghai for Science and Technology, Shanghai 200093, China \\ Correspondence should be addressed to Haibo Wang; nunywanghaibo@126.com \\ Received 3 January 2014; Accepted 3 March 2014; Published 16 March 2014 \\ Academic Editors: F. W. S. Lima and Q.-W. Wang
}

Copyright (C) 2014 Haibo Wang. This is an open access article distributed under the Creative Commons Attribution License, which permits unrestricted use, distribution, and reproduction in any medium, provided the original work is properly cited.

We derive a general expression for the pth power $(p \in N)$ of any complex persymmetric antitridiagonal Hankel (constant antidiagonals) matrices. Numerical examples are presented, which show that our results generalize the results in the related literature (Rimas 2008, Wu 2010, and Rimas 2009).

\section{Introduction}

Solving some difference, differential, and delay differential equations, we meet the necessity to compute the arbitrary positive integer powers of square matrix. Recently, computing the integer powers of antitridiagonal matrices has been a very popular problem. There have been several papers on computing the positive integer powers of various kinds of square matrices by Rimas et al., and others [1-5]. In 2011, the general expression for the entries of the power of complex persymmetric or skew-persymmetric antitridiagonal matrices with constant antidiagonals is presented by GutiérrezGutiérrez [1]. Rimas [2] gave the general expression of the $p$ th power for this type of symmetric odd order antitridiagonal matrices ( $\operatorname{antitridiag}_{n}(1,0,1)$ ) in 2008. In $[3,4]$ a similar problem is solved for antitridiagonal matrices having zeros in main skew diagonal and units in the neighbouring diagonals. In 2010, the general expression for the entries of the power of odd order antitridiagonal matrices with zeros in main skew diagonal and elements $1,1, \ldots, 1 ;-1,-1, \ldots,-1$ in neighbouring diagonals is derived by Rimas [5]. In 2013, Rimas [6] gave the eigenvalue decomposition for real odd order skew-persymmetric antitridiagonal matrices with constant antidiagonals (antitridiag $(a, b, a))$ and derived the general expression for integer powers of such matrices.

In the present paper, we derive a general expression for the $p$ th power $(p \in N)$ of any complex persymmetric antitridiagonal matrices with constant antidiagonals (antitridiag $(b, a, b)$ ). This novel expression is both an extension of the one obtained by Rimas for the powers of the matrix antitridiag $(1,0,1)$ with $n \in N$ (see [2] for the odd case and [5] for the even case) and an extension of the one obtained by Honglin $\mathrm{Wu}$ for the powers of the matrix antitridiag $(1,1,1)$ with $n \in N$ (see [3] for the even case).

\section{Derivation of General Expression}

In this present paper, we study the entries of positive integer power of an $n \times n$ complex persymmetric antitridiagonal matrix with constant antidiagonals as follows:

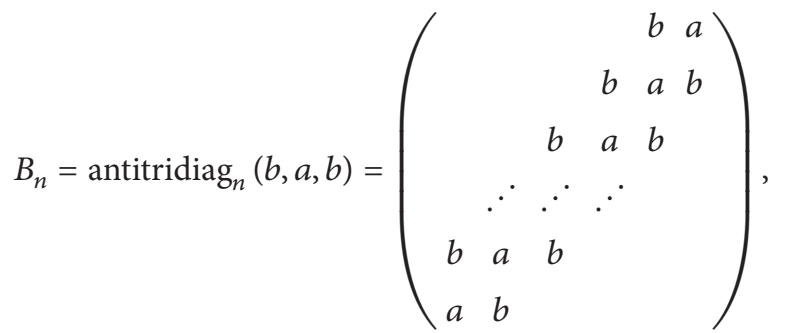

where $a \in C, b \in C \backslash(0)$. 
Consider the following $n \times n$ complex Toeplitz tridiagonal matrix:

$$
A_{n}=\operatorname{tridiag}_{n}(b, a, b)=\left(\begin{array}{cccccc}
a & b & & & & \\
b & a & b & & & \\
& b & a & b & & \\
& & \ddots & \ddots & \ddots & \\
& & & b & a & b \\
& & & & b & a
\end{array}\right) .
$$

The next trivial result relates the matrix $B_{n}$ with $A_{n}$ and with the $n \times n$ backward identity [1]:

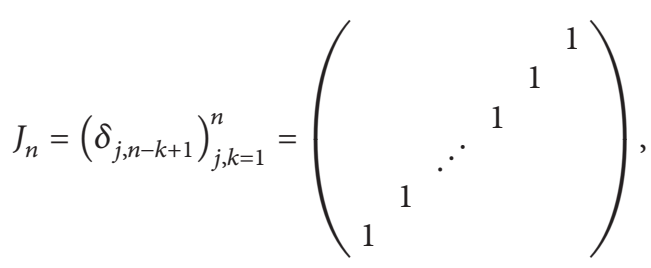

where $\delta$ is the Kronecker delta.

Lemma 1. Let $a \in C, b \in C \backslash(0)$, and $n \in N$. Then

$$
B_{n}=J_{n} A_{n},
$$

where $B_{n}=\operatorname{antitridiag}_{n}(b, a, b)$ and $A_{n}=\operatorname{tridiag}_{n}(b, a, b)$.

Proof. We have

$$
\begin{aligned}
{\left[J_{n} A_{n}\right]_{j, k} } & =\sum_{h=1}^{n}\left[J_{n}\right]_{j, h}\left[A_{n}\right]_{h, k} \\
& =\left[A_{n}\right]_{n+1-j, k} \\
& = \begin{cases}b, & \text { if } n+1-(j+k)=-1, \\
a, & \text { if } n+1-(j+k)=0, \\
b, & \text { if } n+1-(j+k)=1, \\
0, & \text { other. }\end{cases} \\
& =\left[B_{n}\right]_{j, k} .
\end{aligned}
$$

This completes the proof.

We will find the $q$ th power $(q \in N)$ of the matrix (1). Theorem 2 relates all positive integer powers of the matrix $B_{n}$ to $A_{n}$ and $J_{n}$.

Theorem 2. If $a \in C, b \in C \backslash(0)$, and $n \in N$ and if $B_{n}=$ $\operatorname{antitridiag}_{n}(b, a, b)$, then

$$
B_{n}^{q}= \begin{cases}A_{n}^{q}, & \text { if } q \text { is even }, \\ J_{n} A_{n}^{q}, & \text { if } q \text { is odd },\end{cases}
$$

where $A_{n}=\operatorname{tridiag}_{n}(b, a, b)$.

Proof. We will proceed by induction on $q$. The case $q=1$ is obvious.

Suppose that the result is true for $q \geq 1$ and consider case $q+1$.
By the induction hypothesis we have

$$
B_{n}^{q+1}=B_{n} B_{n}^{q}= \begin{cases}B_{n} J_{n} A_{n}^{q}, & \text { if } q+1 \text { is even, } \\ B_{n} A_{n}^{q}, & \text { if } q+1 \text { is odd. }\end{cases}
$$

Since $B_{n}=J_{n} A_{n}$ we obtain that

$$
B_{n}^{q+1}= \begin{cases}J_{n} A_{n} J_{n} A_{n}^{q}, & \text { if } q+1 \text { is even, } \\ J_{n} A_{n}\left(A_{n}^{q}\right), & \text { if } q+1 \text { is odd. }\end{cases}
$$

Since $A_{n}$ is symmetric and $J_{n}^{-1}=J_{n}$, we have

$$
B_{n}^{q}= \begin{cases}A_{n}^{q}, & \text { if } q \text { is even, } \\ J_{n} A_{n}^{q}, & \text { if } q \text { is odd. }\end{cases}
$$

This completes the proof.

Next, we have to solve $A_{n}^{q}$.

We begin this work by reviewing a theorem regarding the Hermitian Toeplitz tridiagonal matrix $A_{n}$.

Theorem 3. Let $a \in C, b \in C \backslash(0)$, and $n \in N$. Then $A_{n}$ has eigenvalues

$$
\lambda_{i}=a+2|b| \cos \frac{i \pi}{n+1}, \quad 1 \leq j \leq n .
$$

Proof. See [7].

With the tridiagonal matrix $A_{n}=\operatorname{tridiag}_{n}(b, a, b)$, we associate the polynomial sequence $\left\{P_{i}\right\}_{-1 \leq i \leq n}$ characterized by a three-term recurrence relation:

$$
\begin{array}{r}
x P_{i}(x)=b P_{i+1}(x)+a P_{i}(x)+b P_{i-1}(x), \\
i=0,1, \ldots, n-1 .
\end{array}
$$

With initial conditions $P_{-1}(x)=0$ and $P_{0}(x)=1$, we can write the relations (11) in matrix form:

$$
x Q_{n-1}(x)=A_{n} Q_{n-1}(x)+P_{n}(x) E_{n},
$$

where $Q_{n-1}(x)=\left[P_{0}(x), P_{1}(x), \ldots, P_{n-1}(x)\right]^{T}$ and $E_{n}=$ $[0,0, \ldots, 0,1]^{T} \in R^{n}$.

Lemma 4. For $i \geq 0$, the degree of the polynomial $P_{i}$ is $i$ and $P_{i}$ and $P_{i+1}$ has no common root.

Proof. See [7].

One can show that the characteristic polynomial of $A_{n}$ is precisely $\left(\Pi_{k=1}^{n} c_{k}\right) P_{n}(x)$. Hence the eigenvalues of $A_{n}$ are exactly the roots of $P_{n}$.

If $\left(\lambda_{j}\right)_{0 \leq j \leq n-1}$ are the roots of the polynomial $P_{n}$, then it follows from (12) that each $\lambda_{j}$ is an eigenvalue of the matrix $A_{n}$ and $Q_{n-1}(x)=\left[P_{0}(x), P_{1}(x), \ldots, P_{n-1}(x)\right]^{T}$ is a corresponding eigenvector $[5,7,8]$. This observation should be taken into account elsewhere in the paper.

The polynomials $\left\{P_{i}\right\}_{0<i<n}$ verify the well-known Christoffel-Darboux Identity. 
Lemma 5. We have

$$
\sum_{i=0}^{n-1} P_{i}(x) P_{i}(y)=\frac{P_{n}(y) P_{n-1}(x)-P_{n}(x) P_{n-1}(y)}{y-x},
$$

for $x \neq y$.

Proof. See [7].

Tending $y$ to $x$ in formula (13), we get

$$
\sum_{i=0}^{n-1} P_{i}(x) P_{i}(x)=P_{n}^{\prime}(x) P_{n-1}(x)-P_{n}(x) P_{n-1}^{\prime}(x) .
$$

Since the matrix $A_{n}$ has distinct eigenvalues $\lambda_{0}, \lambda_{1}$, $\ldots, \lambda_{n-1}$, thus, the eigendecomposition of the matrix $A_{n}$ is

$$
A_{n}=T D T^{-1}
$$

where $D=\operatorname{diag}\left(\lambda_{0}, \lambda_{1}, \ldots, \lambda_{n-1}\right)$ and $T$ is the transforming matrix formed by the eigenvectors of $A_{n}$. Namely, $T=\left(t_{i, j}=\right.$ $\left.P_{i-1}\left(\lambda_{j-1}\right)\right)_{1 \leq i, j \leq n}$, where $\left\{P_{i}\right\}_{-1 \leq i \leq n}$ are defined as above.

Lemma 6. If $T^{-1}=\left(s_{i, j}\right)_{1 \leq i, j \leq n}$, then

$$
s_{i, j}=\frac{P_{j-1}\left(\lambda_{i-1}\right)}{P_{n}^{\prime}\left(\lambda_{i-1}\right) P_{n-1}\left(\lambda_{i-1}\right)} .
$$

Proof. By using the relations (13) and (14), we obtain

$$
\sum_{k=1}^{n} s_{i, k} t_{k, j}=\sum_{k=1}^{n} \frac{P_{k-1}\left(\lambda_{i-1}\right) P_{k-1}\left(\lambda_{j-1}\right)}{P_{n}^{\prime}\left(\lambda_{i-1}\right) P_{n-1}\left(\lambda_{i-1}\right)}=\delta_{i, j},
$$

where $\delta_{i, j}=1$ if $i=j$ and $\delta_{i, j}=0$ if $i \neq j$.

This completes the proof.

For $q \in N$, we have $A_{n}^{q}=T D^{q} T^{-1}$.

We get immediately the following.

Theorem 7. Assume that $q \in N$ and $A_{n}^{q}=\left(\alpha_{i, j}^{(q)}\right)_{1 \leq i, j \leq n}$. Then

$$
\alpha_{i, j}^{(q)}=\sum_{k=1}^{n} \lambda_{k-1}^{q} \frac{P_{i-1}\left(\lambda_{k-1}\right) P_{j-1}\left(\lambda_{k-1}\right)}{P_{n}^{\prime}\left(\lambda_{k-1}\right) P_{n-1}\left(\lambda_{k-1}\right)} .
$$

By using the Cauchy Integral Formula, we can give another expressions of the coefficients $\alpha_{i, j}^{(q)}$ as follows:

$$
\alpha_{i, j}^{(q)}=\int_{C} z^{q} \frac{P_{i-1}(z) P_{j-1}(z)}{P_{n}(z) P_{n-1}(z)} d z,
$$

where $C$ is a closed curve containing the roots of $P_{n}$ and no roots of $P_{n-1}$.

Corollary 8. If the matrix $A_{n}$ is nonsingular with $A^{-1}=$ $\left(\alpha_{i, j}^{(-1)}\right)_{1 \leq i, j \leq n}$, then

$$
\begin{gathered}
A_{n}^{-1}=T D^{-1} T^{-1}, \\
\alpha_{i, j}^{(-1)}=\sum_{k=1}^{n} \frac{1}{\lambda_{k-1}} \frac{P_{i-1}\left(\lambda_{k-1}\right) P_{j-1}\left(\lambda_{k-1}\right)}{P_{n}^{\prime}\left(\lambda_{k-1}\right) P_{n-1}\left(\lambda_{k-1}\right)} .
\end{gathered}
$$

By using the Cauchy Integral Formula, we can give other expressions of the coefficients $\alpha_{i, j}^{(-1)}$ :

$$
\alpha_{i, j}^{(-1)}=\int_{C} z^{-1} \frac{P_{i-1}(z) P_{j-1}(z)}{P_{n}(z) P_{n-1}(z)} d z,
$$

where $C$ is a closed curve containing the roots of $P_{n}$ and no roots of $P_{n-1}$.

Theorem 9. Assume that $q \in N$ and $B_{n}^{q}=\left(\beta_{i, j}^{(q)}\right)_{1 \leq i, j \leq n}$. Then

$$
\beta_{i, j}^{(q)}= \begin{cases}\sum_{k=1}^{n} \lambda_{k-1}^{q} \frac{P_{i-1}\left(\lambda_{k-1}\right) P_{j-1}\left(\lambda_{k-1}\right)}{P_{n}^{\prime}\left(\lambda_{k-1}\right) P_{n-1}\left(\lambda_{k-1}\right)}, & \text { if } q \text { is even, } \\ \sum_{k=1}^{n} \lambda_{k-1}^{q} \frac{P_{n-i}\left(\lambda_{k-1}\right) P_{j-1}\left(\lambda_{k-1}\right)}{P_{n}^{\prime}\left(\lambda_{k-1}\right) P_{n-1}\left(\lambda_{k-1}\right)}, & \text { if } q \text { is odd. }\end{cases}
$$

By using the Cauchy Integral Formula, we can give other expressions of the coefficients $\beta_{i, j}^{(q)}$ :

$$
\beta_{i, j}^{(q)}= \begin{cases}\int_{C} z^{q} \frac{P_{i-1}(z) P_{j-1}(z)}{P_{n}(z) P_{n-1}(z)} d z, & \text { if } q \text { is even, } \\ \int_{C} z^{q} \frac{P_{n-i}(z) P_{j-1}(z)}{P_{n}(z) P_{n-1}(z)} d z, & \text { if } q \text { is odd, }\end{cases}
$$

where $C$ is a closed curve containing the roots of $P_{n}$ and no roots of $P_{n-1}$.

Proof. From Theorem 2 we get

$$
\begin{aligned}
{\left[B_{n}^{q}\right]_{i, j} } & = \begin{cases}{\left[A_{n}^{q}\right]_{i, j},} & \text { if } q \text { is even, } \\
{\left[J_{n} A_{n}^{q}\right]_{i, j}=\sum_{n=1}^{n}\left[J_{n}\right]_{i, h}\left[A_{n}^{q}\right]_{h, j},} & \text { if } q \text { is odd. }\end{cases} \\
& = \begin{cases}{\left[A_{n}^{q}\right]_{i, j},} & \text { if } q \text { is even, } \\
{\left[A_{n}^{q}\right]_{n+1-i, j},} & \text { if } q \text { is odd. }\end{cases}
\end{aligned}
$$

Namely,

$$
\beta_{i, j}^{(q)}= \begin{cases}\alpha_{i, j}^{(q)}, & \text { if } q \text { is even, } \\ \alpha_{n+1-i, j}^{(q)}, & \text { if } q \text { is odd. }\end{cases}
$$

From Theorem 7 it follows that

$$
\beta_{i, j}^{(q)}= \begin{cases}\sum_{k=1}^{n} \lambda_{k-1}^{q} \frac{P_{i-1}\left(\lambda_{k-1}\right) P_{j-1}\left(\lambda_{k-1}\right)}{P_{n}^{\prime}\left(\lambda_{k-1}\right) P_{n-1}\left(\lambda_{k-1}\right)}, & \text { if } q \text { is even, } \\ \sum_{k=1}^{n} \lambda_{k-1}^{q} \frac{P_{n-i}\left(\lambda_{k-1}\right) P_{j-1}\left(\lambda_{k-1}\right)}{P_{n}^{\prime}\left(\lambda_{k-1}\right) P_{n-1}\left(\lambda_{k-1}\right)}, & \text { if } q \text { is odd. }\end{cases}
$$

By using the Cauchy Integral Formula, we can give other expressions of the coefficients $\beta_{i, j}^{(q)}$ :

$$
\beta_{i, j}^{(q)}= \begin{cases}\int_{C} z^{q} \frac{P_{i-1}(z) P_{j-1}(z)}{P_{n}(z) P_{n-1}(z)} d z, & \text { if } q \text { is even, } \\ \int_{C} z^{q} \frac{P_{n-i}(z) P_{j-1}(z)}{P_{n}(z) P_{n-1}(z)} d z, & \text { if } q \text { is odd. }\end{cases}
$$

This completes the proof. 
Corollary 10. Assume that $q \in N$ and $B_{n}^{-1}=\left(\beta_{i, j}^{(-1)}\right)_{1 \leq i, j \leq n}$. Then

$$
\beta_{i, j}^{(-1)}=\sum_{k=1}^{n} \frac{1}{\lambda_{k-1}} \frac{P_{i-1}\left(\lambda_{k-1}\right) P_{j-1}\left(\lambda_{k-1}\right)}{P_{n}^{\prime}\left(\lambda_{k-1}\right) P_{n-1}\left(\lambda_{k-1}\right)} .
$$

By using the Cauchy Integral Formula, we can give other expressions of the coefficients $\beta_{i, j}^{(-1)}$ :

$$
\beta_{i, j}^{(-1)}=\int_{C} z^{(-1)} \frac{P_{i-1}(z) P_{j-1}(z)}{P_{n}(z) P_{n-1}(z)} d z,
$$

where $C$ is a closed curve containing the roots of $P_{n}$ and no roots of $P_{n-1}$.

\section{Numerical Examples}

Consider the $n$ order antitridiagonal matrix $B_{n}$ of the following type:

$$
B_{n}=\left(\begin{array}{cccccc} 
& & & & 1 & a \\
& & & 1 & a & 1 \\
& & 1 & a & 1 & \\
& & \cdot & . & . & \\
1 & a & 1 & & & \\
a & 1 & & & &
\end{array}\right) \text {. }
$$

Assume that

$$
\begin{aligned}
& A_{n}=\left(\begin{array}{cccccc}
a & 1 & & & & \\
1 & a & 1 & & & \\
& 1 & a & 1 & & \\
& & \ddots & \ddots & \ddots & \\
& & & 1 & a & 1 \\
& & & & 1 & a
\end{array}\right),
\end{aligned}
$$

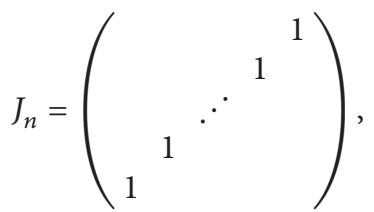

where $A_{n}$ and $J_{n}$ are $n \times n$ matrix. The polynomial sequence $\left\{P_{i}\right\}_{-1 \leq i \leq n}$ verifies

$$
\begin{array}{r}
x P_{i}(x)=P_{i+1}(x)+a P_{i}(x)+P_{i-1}(x), \\
i=0,1, \ldots, n-1,
\end{array}
$$

with initial conditions $P_{-1}(x)=0$ and $P_{0}(x)=1$.

By simple calculation we can show that

$$
P_{i}(x)=U_{i}\left(\frac{x-a}{2}\right), \quad i=0, \ldots, n,
$$

where $U_{i}$ are the Chebyshev polynomials [8] of the second kind which satisfies the three-term recurrence relations:

$$
2 x U_{i}(x)=U_{i+1}(x)+U_{i-1}(x),
$$

with initial conditions $U_{0}(x)=1$ and $U_{1}(x)=2 x$.
Each $U_{n}$ satisfies

$$
U_{n}(x)=\frac{\sin ((n+1) \arccos x)}{\sin (\arccos x)},
$$

and thus the roots of $U_{n}(x)$ are $z_{k}=\cos (k \pi /(n+1)), k=$ $1, \ldots, n$. Then, the eigenvalues of $A$ are

$$
\lambda_{k}=a+2 \cos \frac{k \pi}{n+1}, \quad k=1, \ldots, n .
$$

We get by Theorem 7 the following.

Assume that $q \in N$ and $A_{n}^{q}=\left(\alpha_{i, j}^{(q)}\right)_{1 \leq i, j \leq n}$. Then

$$
\begin{aligned}
& \alpha_{i, j}^{(q)} \\
& =2 \sum_{k=1}^{n}\left(a+2 \cos \frac{k \pi}{n+1}\right)^{q} \\
& \quad \times \frac{U_{i-1}(\cos (k \pi /(n+1))) U_{j-1}(\cos (k \pi /(n+1)))}{U_{n}^{\prime}(\cos (k \pi /(n+1))) U_{n-1}(\cos (k \pi /(n+1)))} .
\end{aligned}
$$
then

If the matrix $A_{n}$ is nonsingular and $A^{-1}=\left(\alpha_{i, j}^{(-1)}\right)_{1 \leq i, j \leq n}$,

$$
\begin{aligned}
& a_{i, j}^{(-1)} \\
& =2 \sum_{k=1}^{n} \frac{1}{a+2 \cos (k \pi /(n+1))} \\
& \quad \times \frac{U_{i-1}(\cos (k \pi /(n+1))) U_{j-1}(\cos (k \pi /(n+1)))}{U_{n}^{\prime}(\cos (k \pi /(n+1))) U_{n-1}(\cos (k \pi /(n+1)))} .
\end{aligned}
$$

We can obtain the following:

$\beta_{i, j}^{(q)}$

$$
=\left\{\begin{array}{r}
2 \sum_{k=1}^{n}\left(a+2 \cos \frac{k \pi}{n+1}\right)^{q} \\
\times \frac{U_{i-1}(\cos (k \pi /(n+1))) U_{j-1}(\cos (k \pi /(n+1)))}{U_{n}^{\prime}(\cos (k \pi /(n+1))) U_{n-1}(\cos (k \pi /(n+1)))} \\
\text { if } q \text { is even, } \\
2 \sum_{k=1}^{n}\left(a+2 \cos \frac{k \pi}{n+1}\right)^{q} \\
\times \frac{U_{n-i}(\cos (k \pi /(n+1))) U_{j-1}(\cos (k \pi /(n+1)))}{U_{n}^{\prime}(\cos (k \pi /(n+1))) U_{n-1}(\cos (k \pi /(n+1)))} \\
\text { if } q \text { is odd }
\end{array}\right.
$$

$\beta_{i, j}^{(-1)}$

$$
\begin{aligned}
=2 \sum_{k=1}^{n}( & \left.a+2 \cos \frac{k \pi}{n+1}\right)^{-1} \\
& \times \frac{U_{i-1}(\cos (k \pi /(n+1))) U_{j-1}(\cos (k \pi /(n+1)))}{U_{n}^{\prime}(\cos (k \pi /(n+1))) U_{n-1}(\cos (k \pi /(n+1)))} .
\end{aligned}
$$


Theorem 11. Consider an odd natural number $n=2 m+1, m \in$ $N$. Let $B_{n}=\operatorname{antitridiag}_{n}(1,0,1)$ and $\lambda_{k}=-2 \cos (k \pi /(n+1))$ for every $1 \leq k \leq n$. Then

$$
\begin{aligned}
{\left[B_{n}^{q}\right]_{i, j}=} & \frac{1+(-1)^{q+i+j}}{2 n+2} \\
& \times \sum_{k=1}^{(n-1) / 2} \beta_{i, j}(k) \lambda_{k}^{q}\left(4-\lambda_{k}^{2}\right) U_{n-i}\left(\frac{\lambda_{k}}{2}\right) U_{n-j}\left(\frac{\lambda_{k}}{2}\right),
\end{aligned}
$$

for all $q \in N$ and $1 \leq i, j \leq n$, where

$$
\beta_{i, j}(k)= \begin{cases}1, & \text { if } i+j \text { is even, } \\ (-1)^{k-1}, & \text { if } i+j \text { is odd. }\end{cases}
$$

$\lambda_{k}(k=1,2, \ldots, n)$ are the eigenvalues of the matrix $B_{n}$ and $U_{k}(x)$ is the kth degree Chebyshev polynomial of the second kind.

Theorem 12. Consider an even natural number $n=2 m, m \in$ $N$. Let $B_{n}=\operatorname{antitridiag}_{n}(1,0,1)$ and $\lambda_{k}=-2 \cos (k \pi /(n+1))$ for every $1 \leq k \leq n$. Then

$$
\left[B_{n}^{q}\right]_{i, j}=\frac{1}{n+1} \gamma_{i, j} \sum_{k=1}^{n / 2} \lambda_{2 k}^{q}\left(4-\lambda_{2 k}^{2}\right) U_{n-i}\left(\frac{\lambda_{2 k}}{2}\right) U_{n-j}\left(\frac{\lambda_{2 k}}{2}\right)
$$

for all $q \in N$ and $1 \leq i, j \leq n$, where

$$
\gamma_{i, j}= \begin{cases}1, & \text { if } i+j \text { is even } \\ 0, & \text { if } i+j \text { is odd }\end{cases}
$$

For even order matrix $B$ the following condition is fulfilled: $\lambda_{k} \neq 0(k=2,4, \ldots, n)$. This means that even order matrix $B_{n}=\operatorname{antitridiag}_{n}(1,0,1)$ is nonsingular (its determinant is not equal to zero) and derived expression of $B_{n}^{q}$ can be applied for computing negative integer powers, as well. Taking $q=-1$, we get the following expression for elements of the inverse matrix $B_{n}^{-1}$ :

$$
\begin{aligned}
{\left[B_{n}^{-1}\right]_{i, j}=} & \frac{1}{n+1} \gamma_{i, j} \sum_{k=1}^{n / 2} \lambda_{2 k}^{-1}\left(4-\lambda_{2 k}^{2}\right) \\
& \times U_{n-i}\left(\frac{\lambda_{2 k}}{2}\right) U_{n-j}\left(\frac{\lambda_{2 k}}{2}\right), \quad i, j=0,1, \ldots, n .
\end{aligned}
$$

Theorem 13. Consider an even natural number $n=2 m$, $m \in N$. Let $B_{n}=\operatorname{antitridiag}_{n}(1,1,1)$ and $\lambda_{k}=(-1)^{k-1}(1+$ $2 \cos (k \pi /(n+1))),(k=1,2, \ldots, n)$. Then

$$
\begin{gathered}
{\left[B_{n}^{q}\right]_{i, j}=\frac{2}{n+1} \sum_{k=1}^{n} \lambda_{k}^{q} \sin \frac{k i \pi}{n+1} \sin \frac{k j \pi}{n+1}} \\
=\frac{2}{n+1} \sum_{k=1}^{n}\left[\lambda_{k}^{q}+(-1)^{i+j}\left(\lambda_{k}+2(-1)^{k}\right)^{q}\right] \\
\times \sin \frac{k i \pi}{n+1} \sin \frac{k j \pi}{n+1} .
\end{gathered}
$$

\section{Conclusion and Discussion}

In this paper, we derive a general expression for the $p$ th power $(p \in N)$ of any complex persymmetric antitridiagonal Hankel (constant antidiagonals) matrices with constant antidiagonals (antitridiag $\left._{n}(b, a, b)\right)$. This novel expression is both an extension of the one obtained by Rimas for the powers of the matrix antitridiag $_{n}(1,0,1)$ with $n \in N$ (see [2] for the odd case and [5] for the even case) and an extension of the one obtained by Honglin $\mathrm{Wu}$ for the powers of the matrix $\operatorname{antitridiag}_{n}(1,1,1)$ with $n \in N$ (see [3] for the even case). We may safely draw the conclusion that our results generalize the results in the related literature $[2,3,5]$.

\section{Conflict of Interests}

The author declares that there is no conflict of interests regarding the publication of this paper.

\section{Acknowledgment}

The author is indebted to the referee for various helpful comments in this paper.

\section{References}

[1] J. Gutiérrez-Gutiérrez, "Powers of complex persymmetric or skew-persymmetric anti-tridiagonal matrices with constant anti-diagonals," Applied Mathematics and Computation, vol. 217, no. 13, pp. 6125-6132, 2011.

[2] J. Rimas, "On computing of arbitrary positive integer powers for one type of symmetric anti-tridiagonal matrices of odd order," Applied Mathematics and Computation, vol. 203, no. 2, pp. 573581, 2008.

[3] H. Wu, "On computing of arbitrary positive powers for one type of anti-tridiagonal matrices of even order," Applied Mathematics and Computation, vol. 217, no. 6, pp. 2750-2756, 2010.

[4] Q. Yin, "On computing of arbitrary positive powers for antitridiagonal matrices of even order," Applied Mathematics and Computation, vol. 203, no. 1, pp. 252-257, 2008.

[5] J. Rimas, "On computing of arbitrary positive integer powers of odd order anti-tridiagonal matrices with zeros in main skew diagonal and elements $1,1,1, \cdots, 1 ;-1,-1,-1, \cdots,-1$ in neighbouring diagonals," Applied Mathematics and Computation, vol. 210, no. 1, pp. 64-71, 2009.

[6] J. Rimas, "Integer powers of real odd order skew-persymmetric anti-tridiagonal matrices with constant anti-diagonals (antitridiag $_{n}(a, c,-a), a \in R \backslash\{0\}, c \in R$," Applied Mathematics and Computation, vol. 219, no. 12, pp. 7075-7088, 2013.

[7] R. A. Horn and C. R. Johnson, Matrix Analysis, Cambridge University Press, New York, NY, USA, 1990.

[8] L. Fox and I. B. Parker, Chebyshev Polynomials in Numerical Analysis, Oxford University Press, London, UK, 1968. 


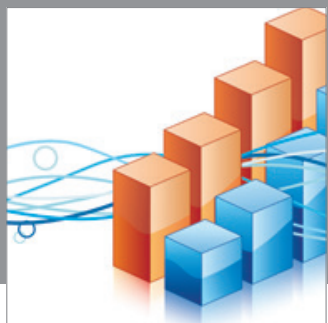

Advances in

Operations Research

mansans

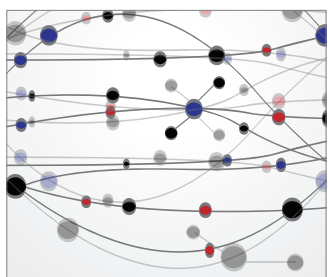

The Scientific World Journal
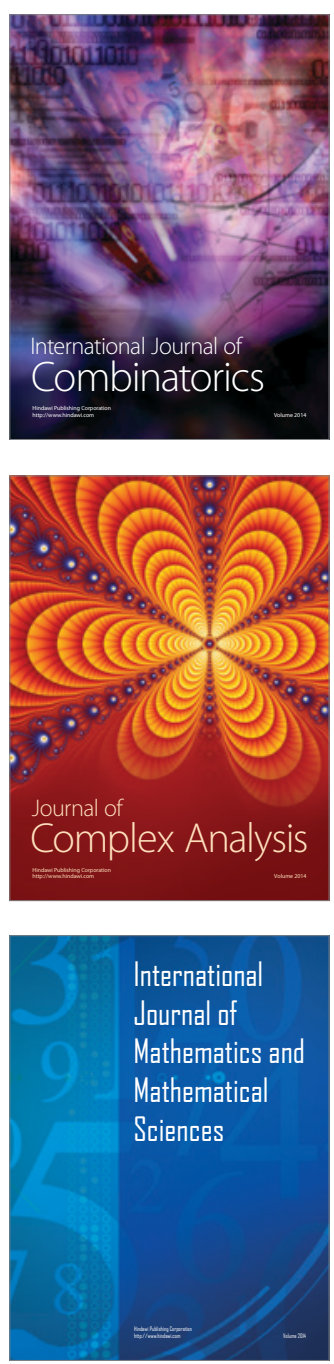
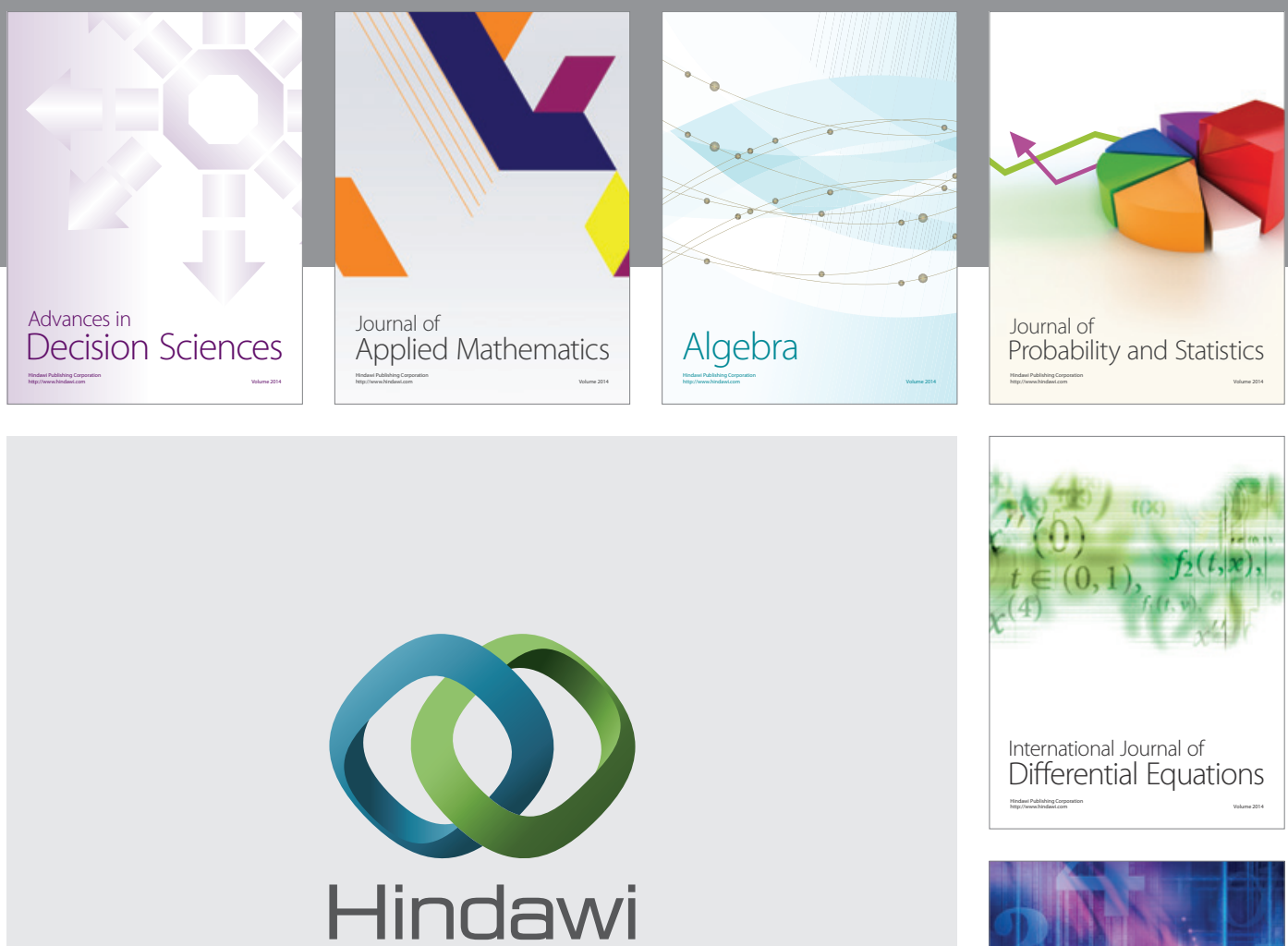

Submit your manuscripts at http://www.hindawi.com
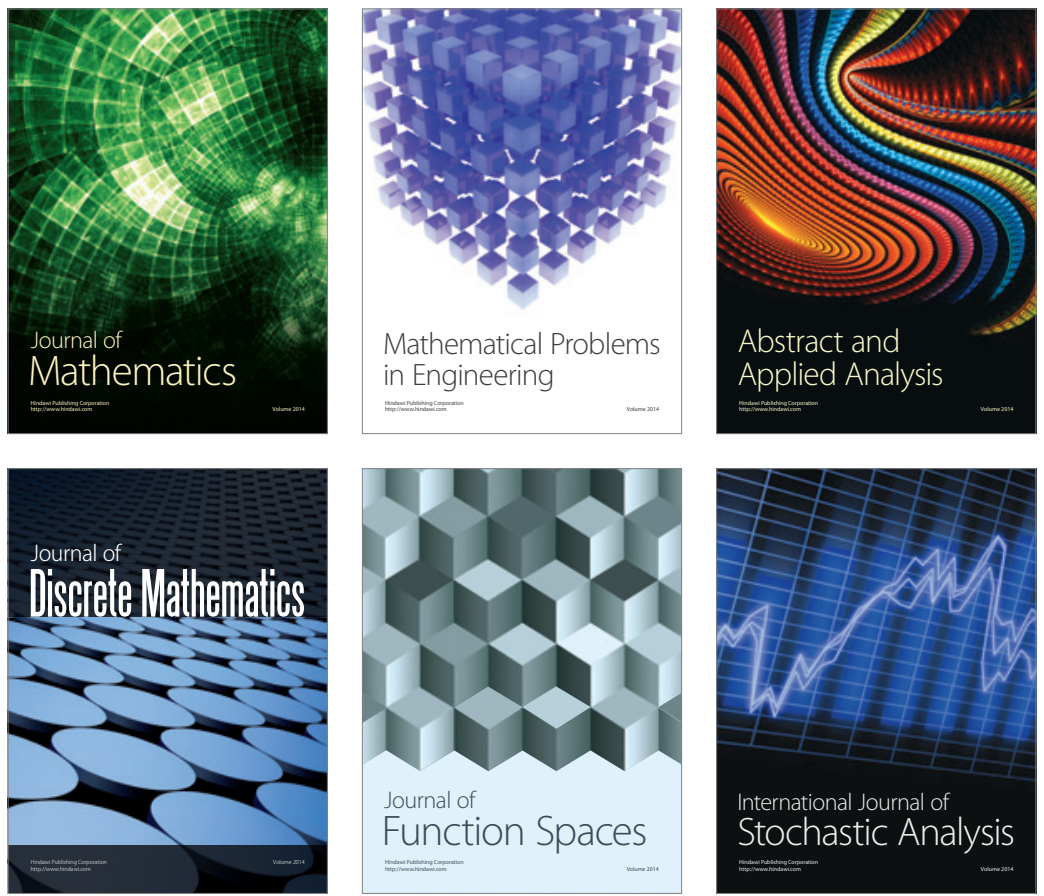

Journal of

Function Spaces

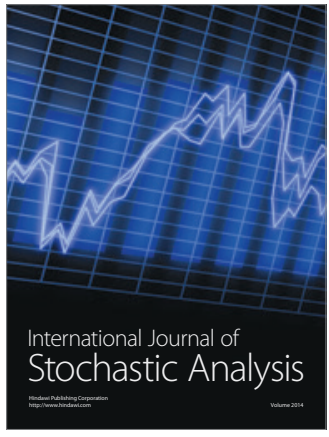

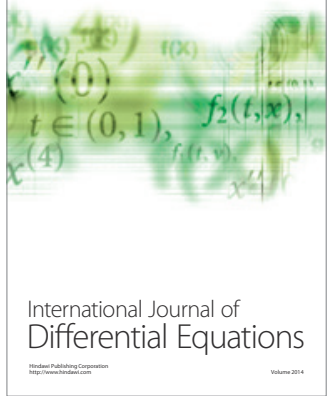
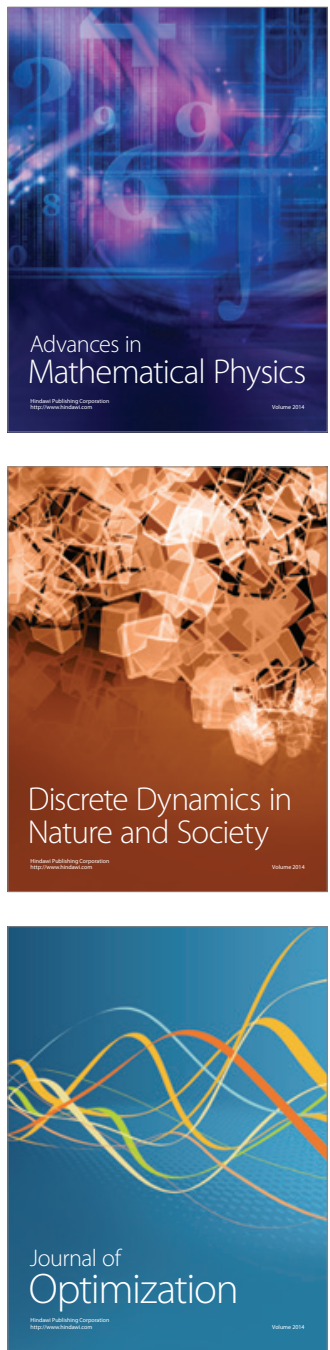